\title{
The Peroxidation of Leukocytes Index Ratio Reveals the Prooxidant Effect of Green Tea Extract
}

\author{
Ilaria Peluso, ${ }^{1}$ Husseen Manafikhi, ${ }^{2}$ Anna Raguzzini, ${ }^{1}$ Yaroslava Longhitano, ${ }^{2}$ \\ Raffaella Reggi, ${ }^{2}$ Christian Zanza, ${ }^{2}$ and Maura Palmery ${ }^{2}$ \\ ${ }^{1}$ Center of Nutrition, Council for Agricultural Research and Economics (CREA-NUT), Via Ardeatina 546, 00178 Rome, Italy \\ ${ }^{2}$ Department of Physiology and Pharmacology "V. Erspamer", "Sapienza" University of Rome, Italy
}

Correspondence should be addressed to Maura Palmery; maura.palmery@uniromal.it

Received 1 November 2016; Accepted 4 December 2016

Academic Editor: Giuseppe Cirillo

Copyright (C) 2016 Ilaria Peluso et al. This is an open access article distributed under the Creative Commons Attribution License, which permits unrestricted use, distribution, and reproduction in any medium, provided the original work is properly cited.

\begin{abstract}
Despite tea increased plasma nonenzymatic antioxidant capacity, the European Food Safety Administration (EFSA) denied claims related to tea and its protection from oxidative damage. Furthermore, the Supplement Information Expert Committee (DSI EC) expressed some doubts on the safety of green tea extract (GTE). We performed a pilot study in order to evaluate the effect of a single dose of two capsules of a GTE supplement $(200 \mathrm{mg} \times 2)$ on the peroxidation of leukocytes index ratio (PLIR) in relation to uric acid (UA) and ferric reducing antioxidant potential (FRAP), as well as the sample size to reach statistical significance. GTE induced a prooxidant effect on leukocytes, whereas FRAP did not change, in agreement with the EFSA and the DSI EC conclusions. Besides, our results confirm the primary role of UA in the antioxidant defences. The ratio based calculation of the PLIR reduced the sample size to reach statistical significance, compared to the resistance to an exogenous oxidative stress and to the functional capacity of oxidative burst. Therefore, PLIR could be a sensitive marker of redox status.
\end{abstract}

\section{Introduction}

The Supplement Information Expert Committee (DSI EC) indicated that consumption of green tea extract (GTE) could induce liver damage [1]. In fact, there are an increasing number of case reports of hepatotoxicity in humans associated with intake of green tea (GT) dietary supplements [2-7]. The types of preparation responsible for the adverse effects were hydroalcoholic extract and aqueous extract of GT, consumed as tea or in capsules [7]. However, there are also cases reporting hepatotoxicity after GT infusion [2-7]. In particular, a case has been reported with features mimicking autoimmune hepatitis, with abnormal liver histology and elevated levels of aspartate aminotransferase, alanine aminotransferase, alkaline phosphatase, gamma glutamyl-transferase and bilirubin, associated with hypergammaglobulinemia, and the transient presence of anti-smooth-muscle antibodies (ASMA) and anti-neutrophil cytoplasmic antibodies (ANCA) [8]. GT withdrawal resulted in a slow and continuous improvement with a complete resolution after 7 months [8].

Furthermore, the Food and Drug Administration (FDA) and the European Food Safety Administration (EFSA) have denied the proposed health claims for GT and decreased risk of noncommunicable diseases [9]. In particular, despite GT increased plasma nonenzymatic antioxidant capacity (NEAC) [10], the EFSA denied claims related to tea and protection of DNA and lipids from oxidative damage [9].

GT contains several flavonoids with antioxidant properties, in particular the flavanol monomers known as catechins, where epigallocatechin-3-gallate (EGCG) is the most effective antioxidant compound [11]. However, tea catechins could have also prooxidant activity [11]. Besides, some of the protective effects of EGCG have been ascribed to its capability to reduce excessive uric acid (UA) level [12]. In particular, flavanols of Camellia sinensis modulate both xanthine oxidase and urate transport [13]. UA is the major plasma antioxidant and contributes to plasma nonenzymatic antioxidant capacity [10].

The peroxidation of leukocytes index ratio (PLIR) measures the resistance of leukocytes to exogenous oxidative stress and their functional capacity of oxidative burst upon activation [14].

Therefore, we performed a pilot study in order to evaluate the effect of a single dose of a GTE supplement on the PLIR, 
in relation to plasma UA and ferric reducing antioxidant potential (FRAP) [15], as well as the sample size to reach statistical significance.

\section{Material and Methods}

2.1. Subjects and Treatment. Participants $(6$ men and 4 women, 19-35 years old) to the study, who volunteered in response to advertisements, were healthy, nonsmokers and were taking no supplements.

For two days prior to each feeding study the subjects followed a low antioxidant diet (washout) by avoiding all fresh fruit, vegetables, tea, coffee, cocoa, fruit juices, and wine.

On the day of the study, after an overnight fast, venous blood samples were collected (in EDTA-tubes) before (T0), 30 minutes (T0.5), and 3 hours (T3) after a single dose of two capsules of a GTE $(200 \mathrm{mg} \times 2)$, commercially available in Italy (cod. 1820, REGISTRO INTEGRATORI https://www.salute.gov.it/imgs/C_17_pagineAree_3668_ listaFile_itemName_1_file.pdf).

2.2. Plasma Uric Acid and TAC. The plasma was separated by centrifugation at $1300 \times \mathrm{g}$ at $4^{\circ} \mathrm{C}$ for $15 \mathrm{~min}$ and stored at $80^{\circ} \mathrm{C}$. Plasma levels of UA were quantified using colorimetric kits (Sentinel CH. SpA, Italy).

Plasma TAC was measured with the FRAP assay [15]. We calculated also the uric acid- independent FRAP (FRAP-UA) as previously described [16], applying the formula:

$$
\text { FRAP-UA }=\text { FRAP } \mu \mathrm{M}-2 \mathrm{UA} \mu \mathrm{M} .
$$

2.3. PLIR Method. After red blood cells' lysis and 4,4difluoro-5-(4-phenyl-1,3-butadienyl)-4-bora-3a,4a-diazas-indacene-3-undecanoic acid (C11-BODIPY, Invitrogen, final concentration $1 \mu \mathrm{M}$ ) staining, leukocytes were treated as previously described $[14,17]$ with phorbol 12-myristate 13-acetate (PMA, Sigma, final concentration $1 \mu \mathrm{g} / \mathrm{mL}$ ), 2,2' azobis(2-methylpropionamidine) dihydrochloride (AAPH, Sigma, final concentration $10 \mathrm{mM}$ ), 6-hydroxy-2,5,7,8tetramethylchroman-2-carboxylic acid (Trolox, Sigma, final concentration $10 \mu \mathrm{M}$ ), PMA $1 \mu \mathrm{g} / \mathrm{mL}+$ Trolox $10 \mu \mathrm{M}$, or AAPH $10 \mathrm{mM}+$ Trolox $10 \mu \mathrm{M}$. After $30 \mathrm{~min}$ at $37^{\circ} \mathrm{C}$ cells were stored in ice, to stop reactions, and rapidly analyzed on an Accuri C6 BD cytometer.

Data acquired on the Accuri C6 was exported in FCS format and analyzed by FCS express software (De Novo Software) to calculate the ratio of oxidation of the probe C11BODIPY (FL1/FL2).

PLIR was calculated as previously described [14, 17], applying the formula:

$$
\text { PLIR }=\frac{(\text { ratio AAPH } \times \text { ratio } \text { PMA Trolox })}{(\text { ratio AAPH Trolox } \times \text { ratio PMA })} .
$$

2.4. Statistics. Statistical analysis, carried out with Friedman RM ANOVA on Ranks, revealed a normal distribution for all markers (Normality Test Shapiro-Wilk and Equal variance test passed).
TABLE 1: Effect of GTE consumption on plasma antioxidant markers and PLIR.

\begin{tabular}{lccc}
\hline & T0 & T0.5 & T3 \\
\hline UA $\mu \mathrm{M}$ & $334.1 \pm 7.4$ & $324.9 \pm 3.9$ & $324.3 \pm 3.7$ \\
FRAP $\mu \mathrm{M}$ & $1061.5 \pm 38.45$ & $1061.48 \pm 52.85$ & $1083.33 \pm 46.78$ \\
FRAP-UA $\mu \mathrm{M}$ & $393.23 \pm 41.63^{*}$ & $411,57 \pm 53.38$ & $434.59 \pm 41,75^{*}$ \\
PLIR L & $2.09 \pm 0.17$ & $2.09 \pm 0.17$ & $2.37 \pm 0.19$ \\
PLIR M & $1.88 \pm 0.13^{*}$ & $1.86 \pm 0.12$ & $2.19 \pm 0.15^{*}$ \\
PLIR G & $1.80 \pm 0.12^{*}$ & $1.90 \pm 0.14$ & $2.17 \pm 0.15^{*}$ \\
\hline
\end{tabular}

Plasma antioxidant markers in samples collected from 10 healthy subjects before (T0), 0.5 (T0.5), and 3 hours (T3) after the consumption of a single dose of two capsules of a green tea extract (GTE) supplement $(200 \mathrm{mg} \times$ 2). UA: uric acid, FRAP: ferric reducing antioxidant potential, FRAP-UA: uric acid-independent FRAP, PLIR: peroxidation of leukocytes index ratio, L: lymphocytes, M: monocytes, and G: granulocytes. RM ANOVA, with time as within-subjects factor, followed by Student-Newman-Keuls post hoc analysis: T3 versus T0: ${ }^{*} p<0.05$.

Therefore, statistical analysis was carried out with repeated measures analysis of variance (RM ANOVA), with time or treatment as within-subjects factors. StudentNewman-Keuls post hoc analysis (all pairwise multiple comparison procedure) was used to isolate differences between groups. Spearman correlation was used to evaluate relationships between variables. All statistical evaluations were performed using the SigmaStat and SigmaPlot software (Jandel Scientific, Inc.).

\section{Results}

3.1. Plasma Uric Acid and TAC. RM ANOVA, with time as within-subjects factor, followed by Student-NewmanKeuls post hoc analysis (all pairwise multiple comparison procedure), revealed that GTE consumption did not affect FRAP values, whereas 3 hours (T3) after treatment both a nonsignificant decrease in UA and a significant increase in FRAP-UA were found (Table 1).

From the difference of means and the standard deviations (power 0.8; alpha 0.05) we calculated a sample size (to reach statistical significance) of 106 for UA.

3.2. PLIR Method. Treatment with GTE significantly increased PLIR of monocytes and granulocytes at T3, whereas a nonsignificant increase was observed for PLIR of lymphocytes (Table 1). We calculated a sample size (to reach statistical significance) of 80 for PLIR of lymphocytes.

Pearson Product Moment Correlation revealed an inverse correlation of UA with PLIR L (CC $=-0.383, p=0.0368)$, PLIR M $(C C=-0.474, p=0.008)$, and PLIR G $(C C=-0.545$, $p=0.001)$ and a direct correlation of FRAP-UA with PLIR $\mathrm{L}(\mathrm{CC}=0.451, p=0.012)$, PLIR $\mathrm{M}(\mathrm{CC}=0.398, p=0.029)$, and PLIR G $(\mathrm{CC}=0.434, p=0.016)$.

3.3. Ratio of Oxidation of the Probe C11-BODIPY. Typical overlay dot plots of the four treatments used for PLIR calculation and ratio of fluorescence (FL1/FL2) on single cells, before GTE consumption and 3 hours after, are presented in Figures 1 and 2, respectively. 

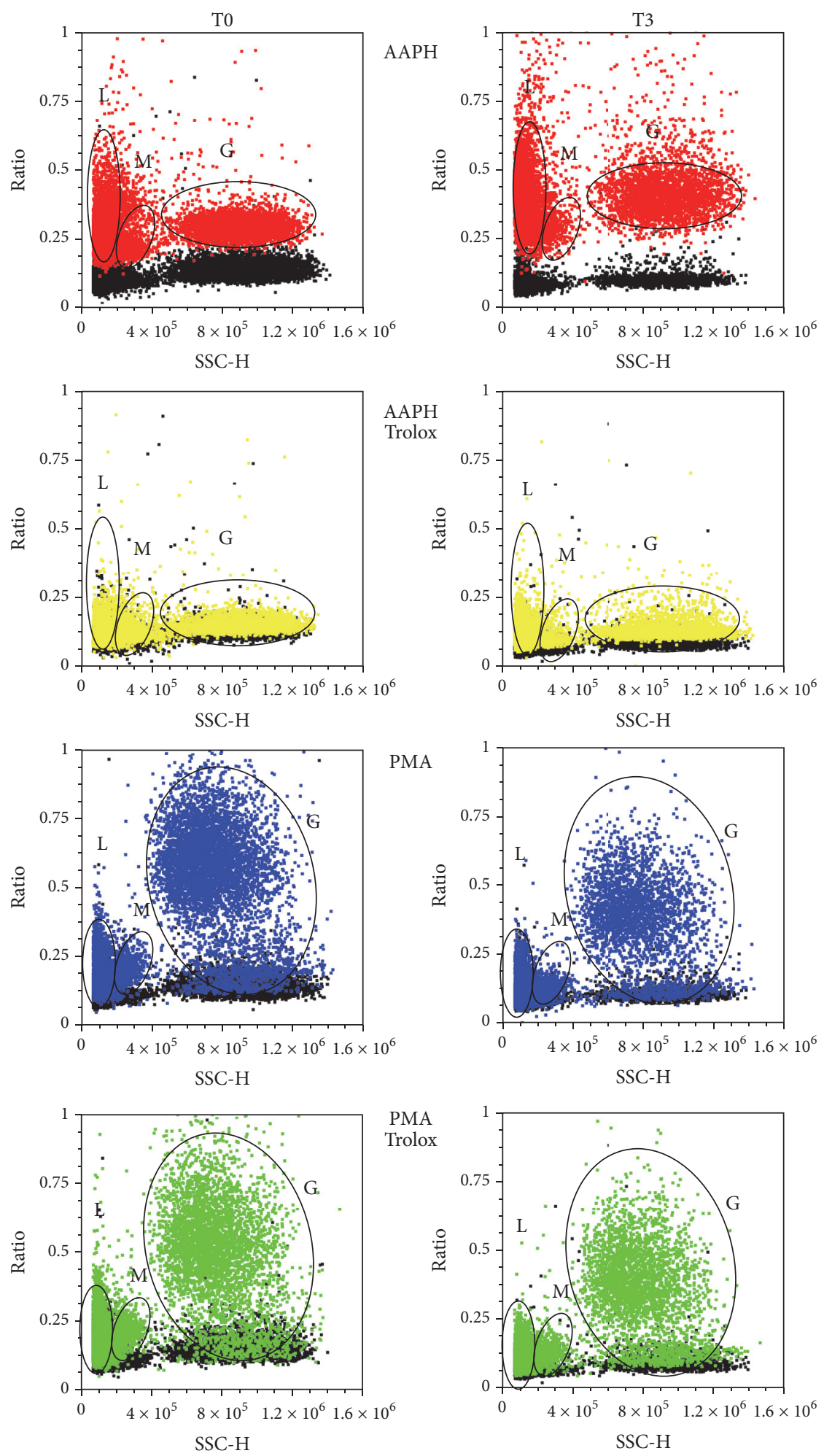

FIGURE 1: Typical overlay dot plots of ratio (ratio of oxidation of the probe C11-BODIPY: FL1/FL2) versus side scatter (SSC): before (T0) and 3 hours (T3) in leukocytes collected after a single dose of two capsules of a green tea extract (GTE) supplement (200 mg $\times 2)$. L: lymphocytes, M: monocytes, and G: granulocytes. Unstimulated samples (black) and leukocytes treated with 2,2' -azobis(2-methylpropionamidine) dihydrochloride (AAPH, $10 \mathrm{mM}$, red), AAPH (10 mM) + 6-hydroxy-2,5,7,8-tetramethylchroman-2-carboxylic acid (Trolox, $10 \mu \mathrm{M}$, yellow), phorbol 12-myristate 13-acetate (PMA, $1 \mu \mathrm{g} / \mathrm{mL}$, blue), or PMA $(1 \mu \mathrm{g} / \mathrm{mL})+$ Trolox (green). 


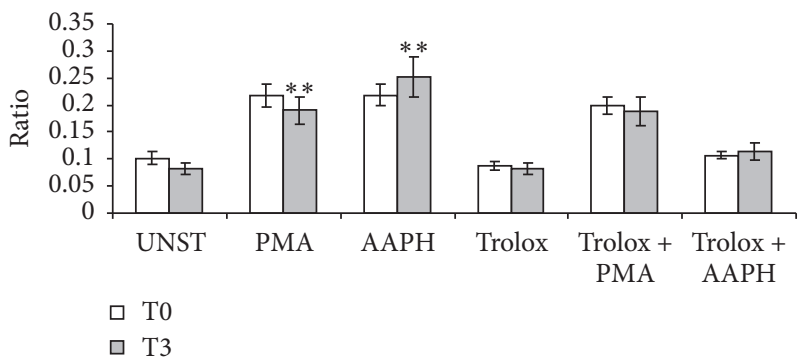

(a)

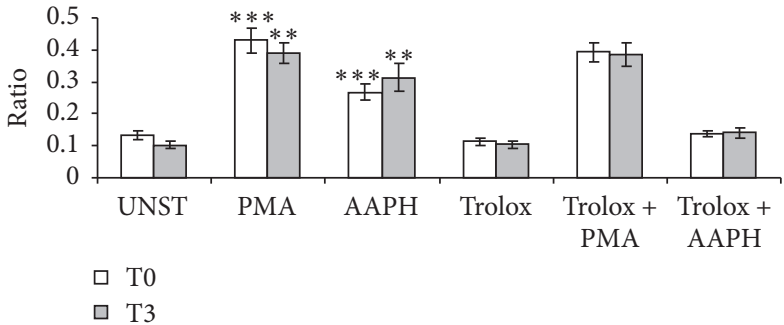

(b)

Figure 2: Ratio (ratio of oxidation of the probe C11-BODIPY: FL1/FL2) of monocytes (a) and granulocytes (b), in samples collected from 10 healthy subjects before (T0) and 3 hours (T3) after the consumption of a single dose of two capsules of a green tea extract (GTE) supplement $(200 \mathrm{mg} \times 2)$. Cells unstimulated $(\mathrm{UNST})$ or treated with phorbol 12 -myristate 13 -acetate $(\mathrm{PMA}, 1 \mu \mathrm{g} / \mathrm{mL}), 2,2^{\prime}$-azobis $(2$ methylpropionamidine) dihydrochloride (AAPH, $10 \mathrm{mM}$ ), 6-hydroxy-2,5,7,8-tetramethylchroman-2-carboxylic acid (Trolox, $10 \mu \mathrm{M})$, Trolox + PMA, or Trolox + AAPH. Two-way (2W) RM ANOVA, with time and treatment as within-subjects factors, followed by Student-NewmanKeuls post hoc analysis: AAPH versus PMA within time: ${ }^{* *} p<0.01,{ }^{* * *} p<0.001$.

Trolox inhibited the peroxidation of C11-BODIPY in leukocytes exposed to AAPH free radicals generating system, but not the PMA-induced oxidation in monocytes and granulocytes, both at $\mathrm{T} 3$ and at T0 (Figure 1).

Considering the major components of PLIR affected by treatment, compared to baseline, the AAPH-induced (exogenous) oxidation appeared greater, whereas the PMAinduced oxidative burst appeared lower (Figure 1).

Despite the differences of ratio PMA and ratio AAPH between times did not reach significance, the statistical significance between ratio AAPH and ratio PMA was different at baseline and at T3 on granulocytes and monocytes (Figure 2). We calculated a sample size (to reach statistical significance between times) of 17 and 33 for ratio AAPH and of 21 and 51 for ratio PMA, for granulocytes and monocytes, respectively.

Ratio PMA was not related to neither UA nor FRAP-UA, whereas ratio AAPH was inversely correlated with UA on all cells (L: $\mathrm{CC}=-0.477, p=0.007 ; \mathrm{M}: \mathrm{CC}=-0.514, p=0.003$; $\mathrm{G}$ : $\mathrm{CC}=-0.511, p=0.003)$, but not with FRAP-UA.

\section{Discussion}

4.1. Effect of GTE on Plasma Antioxidants. Previous studies reported decreased, increased, or unchanged UA and NEAC levels after bolus consumption of EGCG or GTE [1822]. EGCG increased in plasma from $30 \mathrm{~min}$ to 2.6 hours after GTE consumption, depending on the dose and on the formulation [21-25]. However, the FRAP value did not increase when free EGCG concentration was at its peak [21], probably due to the decrease in UA levels observed after GTE consumption [22].

In agreement with these results, in our study GTE consumption did not affect FRAP values whereas a nonsignificant decrease in UA and a significant increase in FRAP-UA were found 3 hours after treatment. The increase in FRAPUA, probably due to the catechins, could counterbalance the reduction in FRAP induced by the UA decrease. However, the FRAP assay matches the antioxidant capacity to the reducing ability [15] and the reduced iron is critical in the onset of oxidative stress due to the Fenton reaction that generates the hydroxyl radical initiator of lipid peroxidation [26].
Therefore, an increase in the metal reducing power could be more likely detrimental than beneficial.

4.2. Effect of GTE on AAPH-Induced Lipoperoxidation. The increase in FRAP-UA was temporally associated with an increased oxidation of the fluorescent probe C11-BODIPY incorporated into the leukocytes. In this context, the prooxidant effects of tea catechins on cells are supported by the molecular mechanisms involved in their induction of antioxidant enzymes, through the antioxidant responsive elements (ARE) pathway $[11,27]$. In particular, it has been suggested that some derivatives of catechins can oxidize highly reactive cysteine thiol groups of Kelch-like ECH-associated protein1 (Keap1), resulting in disulfide bond formation and nuclear factor-erythroid 2-related factor 2 (Nrf2) release [27].

However, the ratio AAPH was inversely correlated with UA on all cells, but not with FRAP-UA. In agreement with our results the consumption for 112 days of a lutein $(12 \mathrm{mg} / \mathrm{d})$ plus GTE $(200 \mathrm{mg} / \mathrm{d})$ supplement did not reduce the oxidation of the C11-BODIY incorporated into the lipid compartment of plasma [28].

4.3. Effect of GTE on PMA-Induced Oxidative Burst. We have found a nonsignificant decrease of PMA-induced lipoperoxidation after GTE consumption, contrarily with the increase of the oxidative burst of granulocyte, observed in cyclists when quercetin was administered with the tea flavanol epigallocatechin 3-gallate, by using dihydrorhodamine 123 (DHR123) as fluorescence probe [29]. However, the post-exerciseinduced decrease in oxidative burst was unaffected after bolus consumption of the same supplement $[29,30]$, when hydroethidine was used as probe [30]. Therefore our results confirm that the plasma membrane C-11 BODIPY is a suitable probe in the evaluation of the effects on the oxidative burst of flavonoids, which increase DHR123 accumulation [31]. Though the effect of GTE consumption on oxidative burst requires more subjects to reach statistical significance, our results are in agreement with the reduction of the p22phox subunit of the NADPH oxidase observed in hemodialysis patients after 6 months of treatment with GT [32]. 
4.4. Effect of GTE on PLIR. In a postprandial study [17], we observed that a functional food covered by dark chocolate and containing glucomannan, inulin, fructooligosaccharides, and Bacillus coagulans strain GanedenBC30 significantly improved postprandial metabolic stress (insulin, glucose, and triglycerides), reduced the postprandial increase of UA, and improved PLIR of lymphocytes, but not of monocytes and granulocytes. We suggested that, although PLIR is a functional index that is independent of baseline levels of oxidation, measuring the ratio between the resistance to exogenous and the resistance to endogenous ROS injury, this ratio calculation could mask the effect of foods that inhibit both the exogenous ROS injury and the oxidative burst [17].

On the contrary, in the present study, treatment with GTE significantly increased PLIR of monocytes and granulocytes at T3 after ingestion, whereas a nonsignificant increase was observed for PLIR of lymphocytes. An inverse correlation of UA with PLIR and a direct correlation of FRAP-UA with PLIR of all leukocytes were found. Therefore, though some of the protective effects of catechins have been ascribed to their capability to reduce excessive UA level [12], in our study the inverse correlation of PLIR with UA levels, in particular with the ratio AAPH component of PLIR, confirms that UA is a major circulating antioxidant as suggested by Fabbrini et al. [33]. Authors [33] reported that rasburicase treatment, in subjects who had high serum UA concentrations, caused a marked decrease in plasma FRAP and a significant increase in urinary isoprostanes/creatinine ratio and in skeletal muscle protein carbonylation.

On the other hand, though the effects of GTE consumption on oxidative burst and AAPH-induced lipoperoxidation require more subjects to reach statistical significance, the statistical significance between ratio AAPH and ratio PMA was different at baseline and at T3 on granulocytes and monocytes. Therefore, the ratio based calculation of the PLIR is able to appreciate differences also with a low number of subjects in monocytes and granulocytes.

\section{Conclusion}

In conclusion our study suggests that PLIR, in particular PLIR of granulocytes where the differences between ratio AAPH and ratio PMA are more evident, reveals the prooxidant effect of GTE. The direct correlation of FRAP-UA with PLIR suggests that the iron reducing power of GTE could be more likely detrimental than beneficial. This result is in agreement with the FDA, the EFSA, and the DSI EC conclusions [1, 9]. Furthermore, the inverse correlation of UA with PLIR confirms the primary role of UA in the antioxidant defences. Therefore, PLIR could be a sensitive marker of redox status.

\section{Abbreviations}

AAPH: $\quad 2,2^{\prime}$-Azobis(2-methylpropionamidine) dihydrochloride

C11-BODIPY: 4,4-Difluoro-5-(4-phenyl-1,3-butadienyl)-4bora-3a,4a-diaza-s-indacene-3-undecanoic acid
DHR123: Dihydrorhodamine 123

DSI EC: Supplement Information Expert Committee

EFSA: European Food Safety Administration

FDA: $\quad$ Food and Drug Administration

FRAP: Ferric reducing antioxidant potential

GT: $\quad$ Green tea

GTE: Green tea extract

NEAC: Nonenzymatic antioxidant capacity

PLIR: Peroxidation of leukocytes index ratio

PMA: Phorbol 12-myristate 13-acetate

Trolox: 6-Hydroxy-2,5,7,8-tetramethylchroman-2carboxylic acid

UA: Uric acid.

\section{Ethical Approval}

Approval for the study was obtained from the Ethics Committee for Human Non-Clinical Research of the Department of Physiology and Pharmacology "V. Erspamer," "Sapienza," University of Rome, and all procedures involving human subjects complied with the Declaration of Helsinki as revised in 2000 .

\section{Consent}

Written informed consent was obtained from all the participants in accordance with the Italian law (law number 196/2003, Ministry of Health Circular Letter GU number $76 / 2008)$.

\section{Competing Interests}

The authors declare that there is no conflict of interests regarding the publication of this paper.

\section{Authors' Contributions}

Ilaria Peluso designed the research, analyzed the data, and drafted the paper. Husseen Manafikhi, Anna Raguzzini, and Raffaella Reggi performed the analyses. Yaroslava Longhitano and Christian Zanza performed the blood sampling. Maura Palmery critically reviewed the paper and supervised the whole project.

\section{Acknowledgments}

The authors thank Fondazione "Enrico ed Enrica Sovena" for the scholarship contribution to Husseen Manafikhi. The authors also thank Claudio Andrew Gobbi for English review of the paper.

\section{References}

[1] D. N. Sarma, M. L. Barrett, M. L. Chavez et al., "Safety of green tea extracts: a systematic review by the US Pharmacopeia," Drug Safety, vol. 31, no. 6, pp. 469-484, 2008.

[2] G. Mazzanti, A. Di Sotto, and A. Vitalone, "Hepatotoxicity of green tea: an update," Archives of Toxicology, vol. 89, no. 8, pp. 1175-1191, 2015. 
[3] F. Stickel, K. Kessebohm, R. Weimann, and H. K. Seitz, "Review of liver injury associated with dietary supplements," Liver International, vol. 31, no. 5, pp. 595-605, 2011.

[4] A. H. Schönthal, "Adverse effects of concentrated green tea extracts," Molecular Nutrition and Food Research, vol. 55, no. 6, pp. 874-885, 2011.

[5] R. Teschke, A. Wolff, C. Frenzel, J. Schulze, and A. Eickhoff, "Herbal hepatotoxicity: a tabular compilation of reported cases," Liver International, vol. 32, no. 10, pp. 1543-1556, 2012.

[6] C. Bunchorntavakul and K. R. Reddy, "Review article: herbal and dietary supplement hepatotoxicity," Alimentary Pharmacology and Therapeutics, vol. 37, no. 1, pp. 3-17, 2013.

[7] C. Di Lorenzo, A. Ceschi, H. Kupferschmidt et al., "Adverse effects of plant food supplements and botanical preparations: a systematic review with critical evaluation of causality," British Journal of Clinical Pharmacology, vol. 79, no. 4, pp. 578-592, 2015.

[8] S. Vanstraelen, J. Rahier, and A. P. Geubel, "Jaundice as a misadventure of a green tea (camellia sinensis) lover: a case report," Acta Gastro-Enterologica Belgica, vol. 71, no. 4, pp. 409412, 2008.

[9] J. T. Dwyer and J. Peterson, "Tea and flavonoids: where we are, where to go next," American Journal of Clinical Nutrition, vol. 98, supplement 6, pp. 1611S-1618S, 2013.

[10] M. Serafini, C. Miglio, I. Peluso, and T. Petrosino, "Modulation of plasma non enzimatic antioxidant capacity (NEAC) by plant foods: the role of polyphenol," Current Topics in Medicinal Chemistry, vol. 11, no. 14, pp. 1821-1846, 2011.

[11] J. D. Lambert and R. J. Elias, "The antioxidant and pro-oxidant activities of green tea polyphenols: a role in cancer prevention," Archives of Biochemistry and Biophysics, vol. 501, no. 1, pp. 6572, 2010.

[12] T. Yokozawa, D. Y. Rhyu, and E. J. Cho, “(-)-Epicatechin 3-O-gallate ameliorates the damages related to peroxynitrite production by mechanisms distinct from those of other free radical inhibitors," Journal of Pharmacy and Pharmacology, vol. 56, no. 2, pp. 231-239, 2004.

[13] I. Peluso, M. Palmery, and A. Vitalone, "Green tea and bone marrow transplantation: from antioxidant activity to enzymatic and multidrug-resistance modulation," Critical Reviews in Food Science and Nutrition, vol. 56, no. 14, pp. 2251-2260, 2016.

[14] I. Peluso, H. Manafikhi, F. Altieri, C. Zanza, and M. Palmery, "The effect of sample storage on the peroxidation of leukocytes index ratio (PLIR) measure," Scientific Reports, vol. 4, article 6539, 2014.

[15] I. F. F. Benzie and J. J. Strain, "The ferric reducing ability of plasma (FRAP) as a measure of 'antioxidant power': the FRAP assay," Analytical Biochemistry, vol. 239, no. 1, pp. 70-76, 1996.

[16] I. Peluso, G. Adorno, A. Raguzzini, L. Urban, A. Ghiselli, and M. Serafini, "A new flow cytometry method to measure oxidative status: the Peroxidation of Leukocytes Index Ratio (PLIR)," Journal of Immunological Methods, vol. 390, no. 1-2, pp. 113-120, 2013.

[17] I. Peluso, H. Manafikhi, R. Reggi, Y. Longhitano, C. Zanza, and M. Palmery, "Relationship between the peroxidation of leukocytes index ratio and the improvement of postprandial metabolic stress by a functional food," Oxidative Medicine and Cellular Longevity, vol. 2016, Article ID 5630985, 10 pages, 2016.

[18] E. Jówko, J. Sacharuk, B. Balasinska et al., "Effect of a single dose of green tea polyphenols on the blood markers of exerciseinduced oxidative stress in soccer players," International Journal of Sport Nutrition and Exercise Metabolism, vol. 22, no. 6, pp. 486-496, 2012.

[19] A. Rabovsky, J. Cuomo, and N. Eich, "Measurement of plasma antioxidant reserve after supplementation with various antioxidants in healthy subjects," Clinica Chimica Acta, vol. 371, no. 1-2, pp. 55-60, 2006.

[20] J. M. M. Van Amelsvoort, K. H. Van Het Hof, J. N. J. J. Mathot, T. P. J. Mulder, A. Wiersma, and L. B. M. Tijburg, "Plasma concentrations of individual tea catechins after a single oral dose in humans," Xenobiotica, vol. 31, no. 12, pp. 891-901, 2001.

[21] M. Kimura, K. Umegaki, Y. Kasuya, A. Sugisawa, and M. Higuchi, "The relation between single/double or repeated tea catechin ingestions and plasma antioxidant activity in humans," European Journal of Clinical Nutrition, vol. 56, no. 12, pp. 11861193, 2002.

[22] S. M. Henning, Y. Niu, Y. Liu et al., "Bioavailability and antioxidant effect of epigallocatechin gallate administered in purified form versus as green tea extract in healthy individuals," Journal of Nutritional Biochemistry, vol. 16, no. 10, pp. 610-616, 2005.

[23] U. Ullmann, J. Haller, J. P. Decourt et al., "A single ascending dose study of epigallocatechin gallate in healthy volunteers," Journal of International Medical Research, vol. 31, no. 2, pp. 88$101,2003$.

[24] M. J. Lee, P. Maliakal, L. Chen et al., "Pharmacokinetics of tea catechins after ingestion of green tea and (-)-epigallocatechin3-gallate by humans: formation of different metabolites and individual variability," Cancer Epidemiology, Biomarkers \& Prevention, vol. 11, no. 10, part 1, pp. 1025-1032, 2002.

[25] H.-H. S. Chow, Y. Cai, D. S. Alberts et al., "Phase I pharmacokinetic study of tea polyphenols following single-dose administration of epigallocatechin gallate and Polyphenon E," Cancer Epidemiology Biomarkers and Prevention, vol. 10, no. 1, pp. 53-58, 2001.

[26] S. Knasmüller, A. Nersesyan, M. Mišík et al., "Use of conventional and -omics based methods for health claims of dietary antioxidants: a critical overview," British Journal of Nutrition, vol. 99, no. 1, pp. ES3-ES52, 2008.

[27] H.-K. Na and Y.-J. Surh, "Modulation of Nrf2-mediated antioxidant and detoxifying enzyme induction by the green tea polyphenol EGCG," Food and Chemical Toxicology, vol. 46, no. 4, pp. 1271-1278, 2008.

[28] L. Li, C.-Y. O. Chen, G. Aldini et al., "Supplementation with lutein or lutein plus green tea extracts does not change oxidative stress in adequately nourished older adults," Journal of Nutritional Biochemistry, vol. 21, no. 6, pp. 544-549, 2010.

[29] D. C. Nieman, D. A. Henson, K. R. Maxwell et al., "Effects of quercetin and egcg on mitochondrial biogenesis and immunity," Medicine and Science in Sports and Exercise, vol. 41, no. 7, pp. 1467-1475, 2009.

[30] M. Konrad, D. C. Nieman, D. A. Henson, K. M. Kennerly, F. Jin, and S. J. Wallner-Liebmann, "The acute effect of ingesting a quercetin-based supplement on exercise-induced inflammation and immune changes in runners," International Journal of Sport Nutrition and Exercise Metabolism, vol. 21, no. 4, pp. 338-346, 2011.

[31] I. Peluso, H. Manafikhi, R. Reggi, and M. Palmery, "Interference of flavonoids with fluorescent intracellular probes: methodological implications in the evaluation of the oxidative burst by flow cytometry," Cytometry A, vol. 85, no. 8, pp. 663-677, 2014. 
[32] U. Vertolli, P. A. Davis, L. Dal Maso et al., "Daily green tea extract supplementation reduces prothrombotic and inflammatory states in dialysis patients," Journal of Functional Foods, vol. 5, no. 3, pp. 1366-1371, 2013.

[33] E. Fabbrini, M. Serafini, I. Colic Baric, S. L. Hazen, and S. Klein, "Effect of plasma uric acid on antioxidant capacity, oxidative stress, and insulin sensitivity in obese subjects," Diabetes, vol. 63, no. 3, pp. 976-981, 2014. 


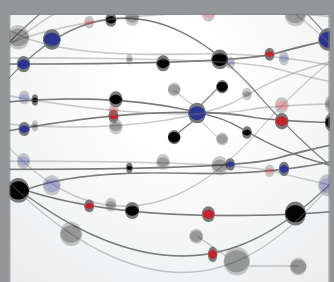

The Scientific World Journal
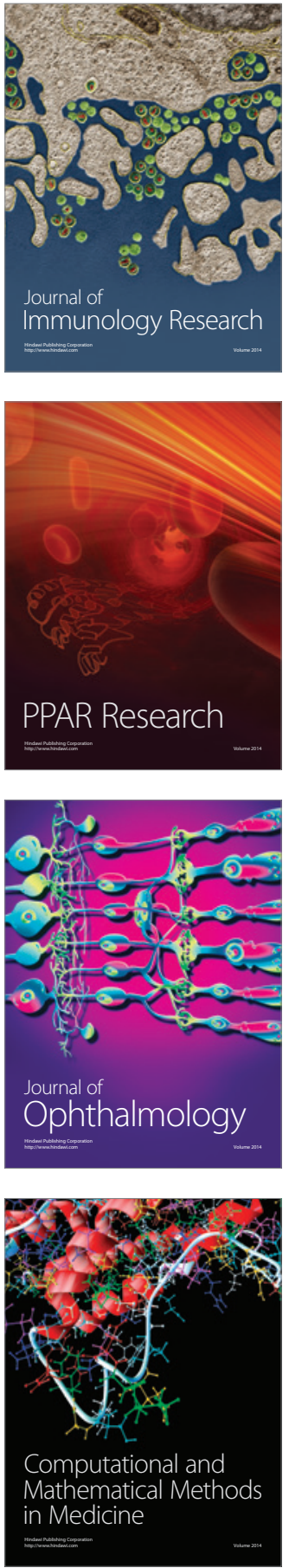

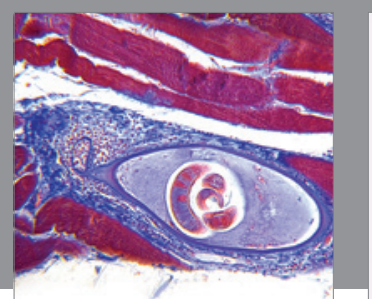

Gastroenterology Research and Practice

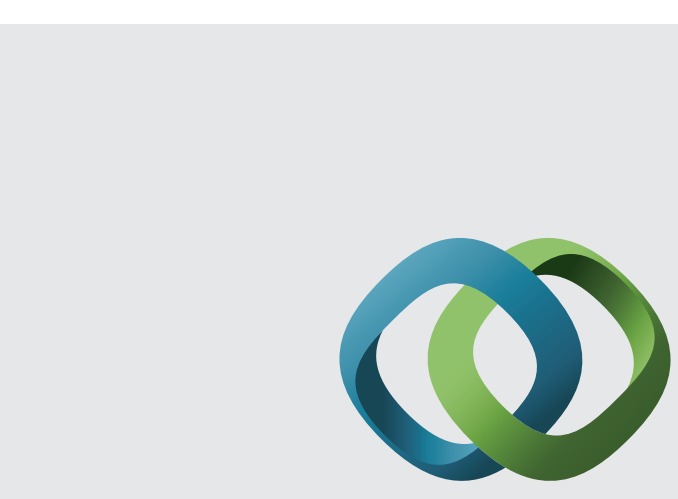

\section{Hindawi}

Submit your manuscripts at

http://www.hindawi.com
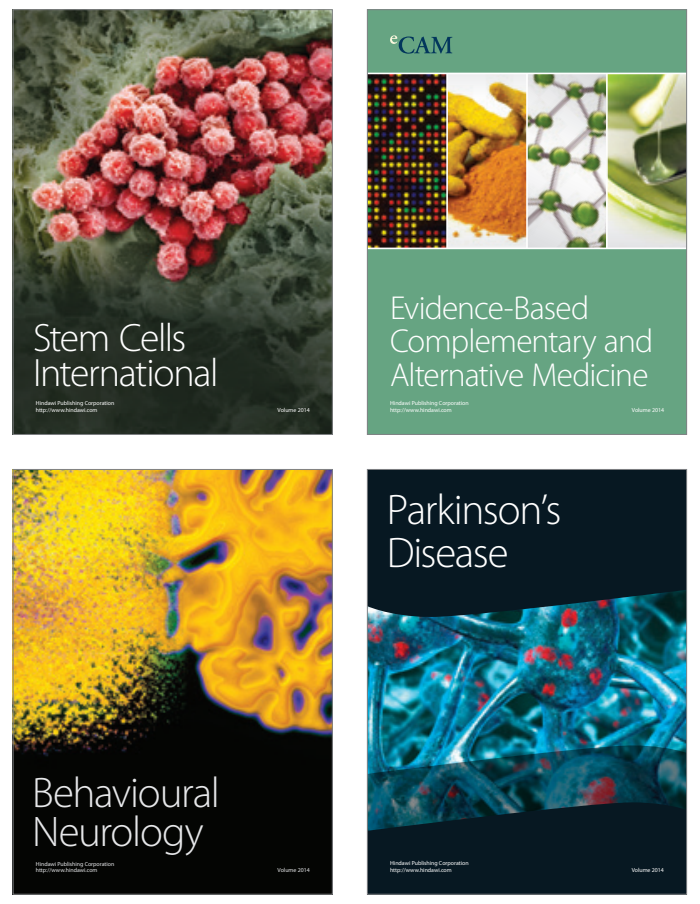
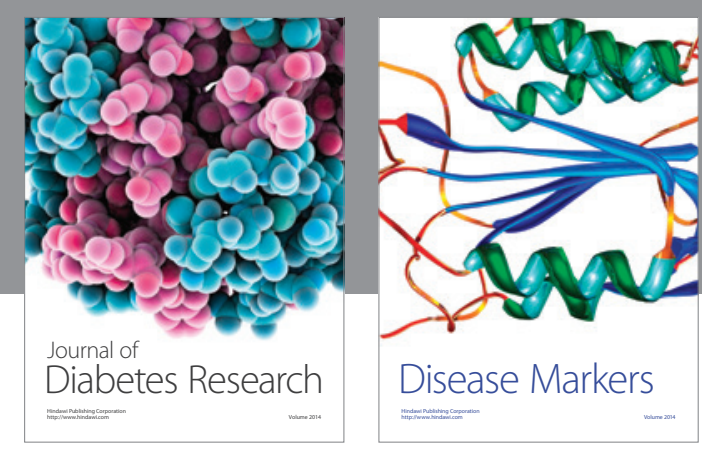

Disease Markers
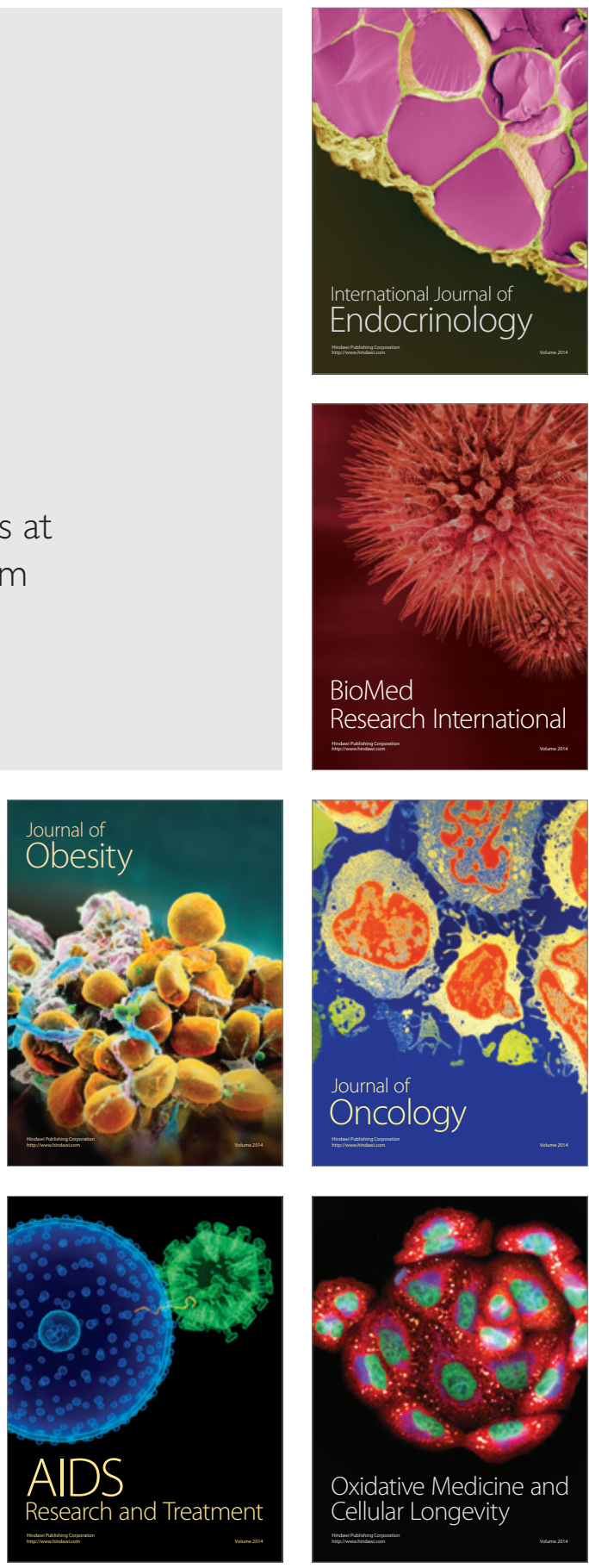